\title{
The Impact of Diffusion and Mixing in Expanding Fluid Drops on The Rate of Surface Tension Change
}

\author{
Orphius I. Mohammad ${ }^{*}$ and Tohren C. G. Kibbey ${ }^{* *}$ \\ School of Civil Engineering and Environmental Science \\ University of Oklahoma \\ 202 W Boyd Street, Rm. 334 \\ Norman, OK 73019 \\ *Current affiliation: Oklahoma Department of Environmental Quality, Oklahoma City, OK
}

Second revision submitted to:

Colloids and Surfaces A: Physicochemical and Engineering Aspects

August 23, 2016

** Corresponding author phone: (405) 325-0580; email: kibbey@ ou.edu. 


\section{Abstract}

2 The dynamics of surface tension change in expanding drops of surfactant solution was

3 studied using drop shape analysis. Experiments explored the dynamics of surface tension change

4 resulting from the flow of surfactant-free water into a surfactant-containing drop. Experiments

5 were conducted for two surfactants, one cationic (cetylpyridinium chloride) and one anionic

6 (sodium octylbenzenesulfonate), both selected because they exhibit rapid adsorption/desorption,

7 allowing for the study of mixing behind the expanding interface. Results were interpreted

8 through the use of a spherical diffusion model designed to simulate drop expansion resulting

9 from influx of surfactant-free water at the core of the drop. Best-fit diffusion constants were

10 found to be consistent with reported molecular diffusion constants for the two surfactants,

11 suggesting that hydraulic mixing within the drop does not play a significant role in the dynamics

12 of surface tension change for the conditions studied. The results of the work have practical

13 implications for applications where interface motion is induced by solute delivery, in that

14 diffusion near the interface is likely to control the dynamics of interfacial tension reduction under

15 many conditions. 


\section{Introduction}

Rate limited adsorption at fluid interfaces has seen extensive study over the years. Many

18 studies have centered around observed dynamic changes in surface or interfacial tension as

19 surface active solutes (surfactants) adsorb at newly created interfaces. Experimental devices

20 such as the bubble pressure tensiometer are capable of measuring interfacial tension in rapidly-

21 formed interfaces, so can provide insight into the dynamics of how surface active solutes adsorb

22 at interfaces [1-3]. Even static measurements of surface tension made using methods like drop

23 shape analysis $[4,5]$ often observe a gradual approach to equilibrium surface tension that occurs

24 over seconds to minutes or longer (e.g., [6, 7]). Rates of change of dynamic surface and

25 interfacial tensions have been observed to vary significantly with different specific surfactants,

26 as well as with different concentrations and other solution conditions [1-3].

A wide range of different models have been used to study dynamic surface tension

28 changes, although most are based on recognition that the observed rate limited behavior is often

29 consistent with the mathematics of diffusion. Ward and Tordai [8] proposed a model based on

30 one-dimensional diffusion (in rectangular coordinates) to- and adsorption on a planar surface

31 from a semi-infinite domain. Their interpretation of experimental data suggested that diffusion

32 in the bulk was likely not the rate limiting process even in the case of very rapid equilibration.

33 Subsequent analysis by Liu and Messow [9] using a more accurate solution to Ward and Tordai's

34 equations came to similar conclusions, suggesting that bulk diffusion plays a significant role only

35 at low concentrations.

36 One weakness with interpretations based on Ward and Tordai's model lies in its use of

37 the one-dimensional diffusion equation in rectangular coordinates, an approach now recognized

38 to yield erroneous interpretations in drops. Models published since Ward and Tordai's work 
39 have taken other diffusion-based approaches. For example, Filippov and Filippova [10]

40 developed equations for adsorption at the interfaces of spherical drops, based on diffusion

41 limited motion in both the bulk and in the adsorption isotherm. Yang and Gu [7] developed a

42 Galerkin finite element solution to solve diffusion and adsorption in a static drop shape defined

43 by a pendant drop profile. In their work they analyzed both the Ward and Tordai [8] and

44 spherical Filippov and Filippova [10] diffusion solutions, concluding that the Ward and Tordai

45 equation worked poorly in a pendant drop, while the predictions of the spherical Filippov and

46 Filippova solution were significantly closer to those determined based on the more accurate

47 geometry used in the finite element solution. Yang and Gu [7] observed dependence on both

48 drop size and syringe needle size in their simulations of adsorption rate, both the result of the

49 impact on the distance over which bulk surfactant diffuses as it is depleted by interfacial

50 adsorption.

51 While substantial efforts have been made in the study of the dynamic processes that

52 underlie dynamic interfacial tensions, little is known about the mixing and diffusion that occurs

53 behind an advancing interface. This is important because many practical applications of surface

54 and interfacial tension modification (for example, subsurface remediation of separate phase

55 organic liquids, oil recovery) depend on using a surface active solute to change the interfacial

56 tension of a moving interface.

57 The focus of the work described here was on examining mixing and diffusion within

58 expanding pendant drops, to understand the impact on the dynamics of the resulting surface

59 tension change. Specifically, drops were formed containing surfactant solution, and then

60 expanded with surfactant-free water to study the influence of mixing and diffusion on the

61 dynamics of the interfacial tension decrease at the surface resulting from dilution within the 
62 drop. Two ionic surfactants were used, one anionic and one cationic. A spherical advection-

63 diffusion model with expanding boundaries was developed to simulate solute behavior in the

64 expanding drops, and used to interpret the results of experiments.

\section{Materials and Methods}

\subsection{Surfactants}

The two surfactants selected for the work were cetylpyridinium chloride (CPC), a

69 cationic surfactant, and sodium octylbenzenesulfonate (SOBS), an anionic surfactant. Both were

70 selected because their adsorption equilibrates at interfaces rapidly, allowing experiments to focus

71 on understanding mixing and diffusion within expanding drops (see the accompanying

72 Supplementary Material section). Both CPC and SOBS were purchased from Sigma-Aldrich (St.

73 Louis, MO). Both surfactants had stated purities of $99 \%$ or higher as received, but were further

74 purified by foam fractionation to remove any highly surface active impurities [11]. Experiments

75 with CPC were conducted without added salt, while experiments with SOBS were conducted in

76 the presence of $0.1 \mathrm{M}$ sodium chloride $(\mathrm{NaCl})$ to increase the efficiency of the highly-

77 hydrophilic SOBS for interfacial tension reduction. All drop expansion experiments were

78 conducted below the CMCs of the two surfactants $\left(9.6 \times 10^{-4} \mathrm{M}\right.$ for $\mathrm{CPC}$ in water, $2.4 \times 10^{-3} \mathrm{M}$ for

79 SOBS in $0.1 \mathrm{M} \mathrm{NaCl})$.

80

\section{2.2. Surface Tension Measurement in Static and Expanding Drops}

All surface tensions were measured using the custom drop shape analysis system and

83 software described in earlier work [12]. Equilibrium measurements of CPC surface tensions

84 were conducted by creating pendant drops with a Cole-Parmer Gilmont (Vernon Hills, IL) $2 \mathrm{~mL}$ 
85 micrometer syringe with a 13 gauge square-end needle, and then imaging the drops over time at

86 approximately $20 \mathrm{sec}$. intervals after an initial brief pause to allow vibrations to dissipate. Three

87 images were collected over the course of approximately one minute, and were analyzed by

88 automated fitting of the Bashforth-Adams equation [12]. Equilibrium data for SOBS in the

89 presence of $0.1 \mathrm{M}$ added $\mathrm{NaCl}$ were taken from previous measurements conducted in our

90 laboratories [13]. No systematic variation in measured surface tension was observed over time

91 for either system in the time scale of the measurements taken.

92 Experiments examining drop expansion were conducted in an enclosed aluminum optical

93 cell with glass windows. The internal dimensions of the cell were $2.5 \mathrm{~cm} \mathrm{high} \times 3 \mathrm{~cm}$ wide $\times 1.5$

$94 \mathrm{~cm}$ deep. The cell was enclosed during the experiment to minimize drop evaporation.

95 Additional water was placed inside the cell to further reduce evaporation. A short section of 13-

96 gauge hypodermic tubing passed through the top of the cell and was connected externally to a

97 syringe pump by way of 1/16" outer diameter PEEK HPLC tubing. Experiments were conducted

98 as follows: surfactant free water (or $0.1 \mathrm{M} \mathrm{NaCl}$ in the case of SOBS experiments) was pumped

99 from the syringe pump to create a small initial drop, typically $0.3-0.5 \mu \mathrm{L}$, as determined by drop

100 shape analysis (Figure 1 A). A $10 \mu \mathrm{L}$ syringe with fine gauge needle was then used to add

101 approximately 5-7 $\mu \mathrm{L}$ of surfactant solution to the drop, expanding its size (Figure $1 \mathrm{~B}$ ). The

102 syringe pump was immediately started, and pure water (or $0.1 \mathrm{M} \mathrm{NaCl}$ in the case of SOBS

103 experiments) was pumped into the drop, causing it to expand (Figures $1 \mathrm{C}-\mathrm{F}$ ). For the work

104 described here, drops were expanded at a rate of approximately $3.9 \mu \mathrm{L} / \mathrm{min}$, as determined from

105 image analysis of expanding drops. Images were captured at 4 second intervals over the course

106 of the experiment. Drops were allowed to expand until they became too large and detached from

107 the hypodermic tubing. It should be noted that experiments like those described here could also 
108 be conducted using a coaxial capillary pendant drop device (e.g., [14-16]) rather than the manual

109 injection method described above (Figure 1B), although the authors are not aware of published

110 reports of its use for that purpose.

111 Evaluations of simulations against equilibrium data were based on comparison with fits

112 to equilibrium surface tension data based on the Szyszkowski Equation (a combination of the

113 Langmuir and Gibbs adsorption equations, Eq. 1) [17].

$$
\Delta \gamma=m R T \Gamma_{\max } \ln (1+K C)
$$

114 where $\Delta \gamma$ is the difference between the surface tension of pure water and the surfactant solution,

$115 \Gamma_{\max }$ is the maximum surface excess of adsorbed surfactant, $K$ is a Langmuir adsorption

116 constant, and $C$ is the surfactant monomer concentration. The multiplier $m$ is either 1 or 2

117 depending on the type of surfactant and amount of added salt (1 for an ionic surfactant with

118 excess salt or for a nonionic surfactant, 2 for an ionic surfactant with no added salt). Fit

119 Szyszkowski equation values for the two surfactants under the conditions studied were: CPC

$120\left(m=2, \Gamma_{\max }=3.09 \times 10^{-6} \mathrm{~mol} / \mathrm{m}^{2}, K=5398 \mathrm{~L} / \mathrm{mol}\right)$, SOBS with $0.1 \mathrm{M} \mathrm{NaCl}\left(m=1, \Gamma_{\max }=4.39 \times 10^{-6}\right.$

$\left.121 \mathrm{~mol} / \mathrm{m}^{2}, K=7178 \mathrm{~L} / \mathrm{mol}\right)$.

123 2.3. Drop Expansion Model

124 Drop expansion was simulated using a diffusion model based on the diffusion equation in

125 spherical coordinates (Eq. 2):

$$
\frac{\partial C}{\partial t}=\frac{D}{r^{2}} \frac{\partial}{\partial r}\left(r^{2} \frac{\partial C}{\partial r}\right)
$$

126 where $C$ is concentration of solute, $t$ is time, and $r$ is distance from the center in a spherical

127 domain. Eq. 1 was solved using a fully implicit shell-centered finite volume formulation [18].

128 Neumann boundary conditions were used in the solution at both $r=0$ (the center of the drop) and 
$129 r=R$ (the outside edge of the drop) to simulate the case of no diffusive flux across the two

130 boundaries. The drop was discretized as concentric shells of equal volume (and thus, with

131 decreasing $\Delta r$ with expanding radius). Advection caused by water entering the center of the drop

132 was simulated by adding additional shells to the outside of the drop at intervals dictated by the

133 flow rate, shifting simulated concentrations outward by one shell, and writing a zero

134 concentration value into the innermost shell - essentially simulating a constant flux of zero

135 concentration water to the center of the drop. Because the shells all have the same volume, mass

136 is conserved when shifting concentrations between shells. Calculated masses were unchanged

137 over the course of simulations to more than 12 significant figures. For all simulations conducted

138 for this work, a shell size of approximately $0.1 \mu \mathrm{L}$ was used, but that size was automatically

139 adjusted slightly for each experiment so that an integral number of shells could exactly represent

140 the starting volume of each drop.

141 Simulations were conducted in Python 2.7 using solvers from the NumPy scientific

142 programming library.

143

\section{3. Results and Discussion}

145 Figure 2 shows the measured surface tension for an expanding CPC drop as a function of

146 the average surfactant concentration in the drop (the total mass of surfactant in the drop divided

147 by its volume). The data in Figure 2 correspond to the same CPC system for which images are

148 shown in Figure 1, i.e., a starting concentration of $3.1 \times 10^{-4} \mathrm{M}$, initially diluted by adding $6.1 \mu \mathrm{L}$

149 of $3.4 \times 10^{-4} \mathrm{M}$ solution to a $0.36 \mu \mathrm{L}$ drop of water (Figures $1 \mathrm{~A}, \mathrm{~B}$ ). Letters $\mathrm{C}, \mathrm{D}, \mathrm{E}$ and F in Fig.

1502 correspond to the image subfigures in Figure 1. Note that when surfactant is added to the drop

151 (C) there is an initial rapid decrease in surface tension, resulting from the mixing that occurs as a 
152 result of the rapid initial drop expansion and corresponding adsorption of the added surfactant.

153 After approximately 30 seconds (the rightmost 7 red circles) the measured surface tension

154 reaches a minimum near $56 \mathrm{mN} / \mathrm{m}$ where it remains for an additional approximately 30 seconds

155 as the drop continues to expand, and then (point D) surface tension begins increasing

156 monotonically with further expansion as the concentration in the drop is further diluted by

157 incoming pure water. Of perhaps greatest interest in Figure 2 is the fact that the observed

158 relationship between surface tension and average concentration do not follow the equilibrium

159 surface tension data, despite the fact that the drop was expanded very slowly (the elapsed time

160 between points $\mathrm{C}$ and $\mathrm{F}$ (Figs. 1, 2) was 6.4 minutes for a volume change of $24.7 \mu \mathrm{L}$ ).

161 Figure 3 shows average concentrations over time for CPC drops with five different

162 starting concentrations. The trends in Figure 3 are parallel to those in Figure 2, and in all cases,

163 the observed curves do not follow the equilibrium surface tension curves.

164 The results in Figures 2 and 3 strongly suggest that despite the slow flow rate and small

165 distances between the drop core and surface, the contents of the drops are not completely mixed.

166 Note that CPC itself adsorbs rapidly at interfaces; surface tension of a new drop formed with a

167 CPC solution equilibrates within the time it takes for the drop to stabilize (seconds).

168 Furthermore, gradually expanding a CPC drop without changing its concentration (i.e.,

169 expanding it by pumping additional solution rather than pure water) produces no observable

170 change in surface tension. As such, the results in the figure are consistent with a case where the

171 concentration at the core of the drop is lower than the concentration at the drop surface, and, as a

172 result, the surface tension is lower than would be predicted based on average surfactant

173 concentration alone. 
To better quantify and understand this phenomenon, simulations were conducted based

175 on the spherical diffusion model described in Sec. 2.3. The simulations started with an initial

176 CPC concentration in the drop calculated from the initial dilution of added surfactant (i.e., Fig. 1

177 A, B). For purposes of this work adsorption at the interface was assumed to be instantaneous, in 178 complete equilibrium with surfactant in the outer shell in the model. Application of the model

179 involved matching measured interfacial tensions over time with the simulated surfactant

180 concentration at the surface at the same times and volumes for a given diffusion constant, $D$.

Figure 4 illustrates the model for a starting CPC concentration of $3.1 \times 10^{-4} \mathrm{M}-$ the same

182 concentration used in Figs. 1 and 2. For large $D$ values (e.g., $10^{-8} \mathrm{~m}^{2} / \mathrm{s}$ in the figure) the

183 surfactant in the drop approaches completely-mixed, and the curves are close to the average

184 concentration curve. (Note that $D$ values of $10^{-7} \mathrm{~m}^{2} / \mathrm{s}$ or larger (not shown) produce surface

185 concentrations essentially equivalent to complete mixing.) For smaller $D$ values, the extent of

186 mixing in the drop during expansion decreases, shifting the curves in Figure 4 to the right. That

187 is, small $D$ values cause concentrations at the surface to change more slowly, remaining high as

188 the drop expands. If $D$ is sufficiently small (e.g., $10^{-11} \mathrm{~m}^{2} / \mathrm{sec}$ in the figure), sufficiently little

189 mixing occurs that the surface concentration in the drop remains essentially constant, yielding a

190 vertical curve. In between these extremes, there is a value of $D$ - in this case $3.37 \times 10^{-10} \mathrm{~m}^{2} / \mathrm{s}-$

191 that corresponds to a curve that closely matches the equilibrium curve. The match between the

192 simulated and equilibrium curves is an indication that the corresponding $D$ accurately describes

193 the extent of mixing that occurs within the drop.

194 Figure 5 shows simulation results for the five CPC concentrations in Figure 3 at a single

$195 D$ of $3.37 \times 10^{-10} \mathrm{~m}^{2} / \mathrm{s}$. This value of $D$ was determined to provide the least-squares best fit

196 between the simulated surface concentrations and concentrations calculated from the 
197 Szyszkowski equation for the experimentally measured surface tensions. (Note that error was

198 minimized in the $\log$ concentration scale.) Note that the magnitude of $D$ determined through this

199 fit is very similar to, and slightly smaller than, the molecular diffusion constants of $6.3 \times 10^{-10}$

$200 \mathrm{~m}^{2} / \mathrm{s}$ and $8.4 \times 10^{-10} \mathrm{~m}^{2} / \mathrm{s}$ reported by Verma et al. [19] and Leaist [20], respectively, for CPC

201 monomers, although slightly larger than the $1.21 \times 10^{-10} \mathrm{~m}^{2} / \mathrm{s}$ suggested by the calculations of

202 Atkin et al. [21]. The similarity is a strong indication that the primary mode of mixing within the

203 expanding drop is molecular diffusion rather than hydraulic mixing induced by the flow of water

204 into the drop. If an appreciably higher value of $D$ were required to simulate the data (e.g., an

205 unrealistically large value for a molecular diffusion constant, such as $10^{-8}$ or $10^{-9} \mathrm{~m}^{2} / \mathrm{s}$ ) it would

206 be a strong indication that additional mixing was occurring within the drop.

207 It is important to emphasize that this result (i.e., mixing controlled by molecular

208 diffusion) might not be expected to hold under conditions of extremely rapid interface expansion,

209 where internal hydraulic mixing would almost certainly contribute to more rapid equilibration

210 than would be predicted from diffusion alone. Additional factors, such the syringe diameter and

211 resulting inflow velocity could also potentially impact the nature of mixing in the drop. In the

212 context of this work, the inlet water had a velocity of approximately $1.5 \mathrm{~mm} / \mathrm{min}$, and the radial

213 expansion velocity of the outer drop surfaces ranged from approximately 0.1 to $0.2 \mathrm{~mm} / \mathrm{min}$,

214 depending on the size of the drop. (Note that the coaxial dye mixing experiments reported by

215 Ferri et al. [16] involved an exchange flow rate greater than $26 \mu \mathrm{L} / \mathrm{min}$, approximately 7 times

216 the volumetric flow rate used in the drop expansion experiments in this study.)

217 While faster flow could potentially be expected to induce a transition from diffusion-

218 dominated mixing to hydrodynamic mixing, it is interesting to consider the implications of the

219 result for even slower expansion than was studied here. It is reasonable to expect that there is a 
220 rate below which an expanding drop can be considered completely mixed, i.e., the concentration 221 can be considered uniform throughout. However, simulations indicate that that rate is

222 exceedingly small. For example, for expansion of a CPC solution, simulations indicate that a

$2231000 \times$ smaller volumetric flow rate (e.g., to $0.0039 \mu \mathrm{L} / \mathrm{min}$ ) would be needed to create

224 conditions where the average concentration in the drop remained within $1 \%$ of the concentration

225 adjacent to the surface. The small magnitude of that flow rate means that for all practical

226 purposes, formation of interfaces caused by slow expansion will result in concentration

227 gradients.

228 Figure 6 shows the spatial distribution of CPC concentrations within the drop, predicted

229 by simulation with $D=3.37 \times 10^{-10} \mathrm{~m}^{2} / \mathrm{s}$. The four plots shown correspond to the four drop

230 images in Figures 1 C, D, E and F. It is apparent from the Figure that even for the very slow

231 expansion rate used (approximately $3.9 \mu \mathrm{L} / \mathrm{min}$ ), much of the surfactant mass remains near the

232 outer surface of the drop during the process of expansion, and the inner core of the drop is

233 essentially pure water.

234 Figure 7 shows expansion data for five different starting concentrations of SOBS in the

235 presence of $0.1 \mathrm{M} \mathrm{NaCl}$. As was observed with the drops containing CPC (Figure 3), the curves

236 of average concentration vs. interfacial tension in Figure 7 remain below the equilibrium curve

237 over the course of drop expansion, an indication that complete mixing is not occurring within the 238 drop.

Figure 8 shows best fit simulation data for the SOBS system. In the case of SOBS, a

240 value of $D=4.50 \times 10^{-10} \mathrm{~m}^{2} / \mathrm{s}$ provides the best fit to the equilibrium data. This value agrees

241 favorably with the value of $6 \times 10^{-10} \mathrm{~m}^{2} / \mathrm{s}$ reported by Lindman et al. [22] for SOBS monomer 
242 diffusion, suggesting that, as was the case with CPC, molecular diffusion is likely the primary

243 mixing mechanism in the expanding SOBS drops.

\section{4. Conclusions}

The results of this work suggest that molecular diffusion controls mixing within slowly-

247 expanding aqueous drops; hydrodynamic mixing caused by inflow of water does not appear to be

248 a significant factor in the dynamics of drop surface tension equilibration. Despite the small

249 distances involved and slow expansion rates, an assumption of complete mixing within the drop

250 provides a very poor prediction of surface tension dynamics.

The significance of this result is that for any application where interface motion results

252 from flow, interfacial behaviors may not be well predicted by fluid properties small distances -

253 even $\mathrm{mm}$ - from the interface, but rather may be limited by bulk diffusion. While conditions of

254 rapid expansion may involve some hydrodynamic mixing which would be more rapid than

255 diffusion-limited mixing, a range of flow rates covering several orders of magnitude exists for

256 which complete mixing behind an interface cannot be practically assumed. This phenomenon

257 may be important in any application that depends on interaction of a solute with a moving fluid

258 interface. It is worth noting that this effect could potentially be exacerbated in systems with

259 higher concentration micellar surfactant solutions, because the diffusion coefficients for micelles

260 can be an order of magnitude or more lower than those of monomers. However, this effect could

261 to some extent be mitigated by the use of concentrations orders of magnitude above the CMC,

262 increasing the driving force for diffusion. 
266 Acknowledgments. This material is based upon work supported by the National Science 267 Foundation under Grant No. 0092995. 


\section{Figure Captions}

Figure 1. Selected images from drop formation and expansion for aqueous CPC. Added CPC concentration $3.3 \times 10^{-4} \mathrm{M}$. Water flow rate $=3.87 \mu \mathrm{L} / \mathrm{min}$.

Figure 2. Measured surface tension $(\gamma)$ vs. average concentration in an expanding drop for the CPC system shown in Figure 1. Letters correspond to subfigures of the corresponding images in Figure 1. Equilibrium data and Szyszkowski equation fit to equilibrium data are also shown for comparison.

Figure 3. Measured surface tension $(\gamma)$ vs. average concentration in expanding drops for five different starting CPC concentrations. Equilibrium data and Szyszkowski equation fit to equilibrium data are also shown for comparison.

Figure 4. Measured surface tension $(\gamma)$ vs. calculated surface concentration in an expanding drop for the CPC system shown in Figures 1 and 2. Calculated surface concentrations are shown for different $D$ values.

Figure 5. Measured surface tension $(\gamma)$ vs. calculated surface concentration in expanding drops for five different starting CPC concentrations. Calculated surface concentrations correspond to $D=3.37 \times 10^{-10} \mathrm{~m}^{2} / \mathrm{sec}$, the least-squares best-fit value for the data.

Figure 6. Calculated spatial configuration of CPC for the system in Figures 1 and 2, based on $D$ $=3.37 \times 10^{-10} \mathrm{~m}^{2} / \mathrm{sec}$. Darker shading corresponds to higher concentration. (Black = $3.1 \times 10^{-4} \mathrm{M}$, White $\left.=0 \mathrm{M}\right)$. Letters correspond to drop images in Figure 1 and data points in Figure 2.

Figure 7. Measured surface tension $(\gamma)$ vs. average concentration in expanding drops for five different starting SOBS concentrations. Equilibrium data and Szyszkowski equation fit to equilibrium data are also shown for comparison. *Equilibrium data from Chen and Kibbey, 2006.

Figure 8. Measured surface tension $(\gamma)$ vs. calculated surface concentration in expanding drops for five different starting SOBS concentrations. Calculated surface concentrations correspond to $D=4.50 \times 10^{-10} \mathrm{~m}^{2} / \mathrm{sec}$, the least-squares best-fit value for the data. 
298 [1] X.Y. Hua, M.J. Rosen, Dynamic Surface-Tension of Aqueous Surfactant Solutions .1. Basic 299 Parameters, J Colloid Interf Sci, 124 (1988) 652-659.

300 [2] Y.H. Xi, M.J. Rosen, Dynamic Surface-Tension of Aqueous Surfactant Solutions .3. Some 301 Effects of Molecular-Structure and Environment, J Colloid Interf Sci, 141 (1991) 180-190.

302 [3] P. Joos, J.P. Fang, G. Serrien, Comments on Some Dynamic Surface-Tension Measurements by the Dynamic Bubble Pressure Method, J Colloid Interf Sci, 151 (1992) 144-149.

[4] Y. Rotenberg, L. Boruvka, A.W. Neumann, Determination of Surface-Tension and Contact306183.

307 [5] S.M.I. Saad, Z. Policova, E.J. Acosta, A.W. Neumann, Range of Validity of Drop Shape

308 Techniques for Surface Tension Measurement, Langmuir, 26 (2010) 14004-14013.

309 [6] S.S. Datwani, K.J. Stebe, Surface tension of an anionic surfactant: Equilibrium, dynamics, 310 and analysis for Aerosol-OT, Langmuir, 17 (2001) 4287-4296.

311 [7] C.D. Yang, Y.G. Gu, Modeling of the adsorption kinetics of surfactants at the liquid-fluid 312 interface of a pendant drop, Langmuir, 20 (2004) 2503-2511.

313 [8] A.F.H. Ward, L. Tordai, Time-Dependence of Boundary Tensions of Solutions .1. The Role 314 of Diffusion in Time-Effects, J Chem Phys, 14 (1946) 453-461.

315 [9] J. Liu, U. Messow, Diffusion-controlled adsorption kinetics at the air/solution interface, 316 Colloid Polym Sci, 278 (2000) 124-129.

317 [10] L.K. Filippov, N.L. Filippova, Dynamic surface tension and adsorption kinetics in finite 318 systems, J Colloid Interf Sci, 187 (1997) 352-362.

319 [11] S.S. Dukhin, G. Kretzschmar, R. Miller, Dynamics of adsorption at liquid interfaces :

320 Theory, expeiment, application, Elsevier, Amsterdam, 1995.

321 [12] O.I. Mohammad, T.C.G. Kibbey, Dissolution-induced contact angle modification in dense 322 nonaqueous phase liquid/water systems, Environ Sci Technol, 39 (2005) 1698-1706.

323 [13] L.X. Chen, T.C.G. Kibbey, Measurement of air-water interfacial area for multiple hysteretic 324 drainage curves in an unsaturated fine sand, Langmuir, 22 (2006) 6874-6880.

325 [14] M.A. Cabrerizo-Vilchez, H.A. Wege, J.A. Holgado-Terriza, A.W. Neumann, Axisymmetric 326 drop shape analysis as penetration Langmuir balance, Rev Sci Instrum, 70 (1999) 2438-2444.

327 [15] H.A. Wege, J.A. Holgado-Terriza, A.W. Neumann, M.A. Cabrerizo-Vilchez, Axisymmetric 328 drop shape analysis as penetration film balance applied at liquid-liquid interfaces, Colloids Surf 329 A, 156 (1999) 509-517. 
330 [16] J.K. Ferri, A.D. Cramer, C. Kotsmar, R. Miller, Coaxial Capillary Pendant Drop 331 Experiments with Subphase Exchange, in: R. Miller, L. Liggieri (Eds.) Bubble and Drop 332 Interfaces, CRC Press, Boca Raton, 2011, pp. 195-221.

333 [17] M.J. Rosen, J.T. Kunjappu, Surfactants and interfacial phenomena, 4th ed., Wiley, 334 Hoboken, N.J., 2012.

335 [18] Y. Zeng, P. Albertus, R. Klein, N. Chaturvedi, A. Kojic, M.Z. Bazant, J. Christensen, 336 Efficient Conservative Numerical Schemes for 1D Nonlinear Spherical Diffusion Equations with 337 Applications in Battery Modeling, J Electrochem Soc, 160 (2013) A1565-A1571.

338 [19] R. Verma, A. Mishra, K.R. Mitchell-Koch, Molecular Modeling of Cetylpyridinium 339 Bromide, a Cationic Surfactant, in Solutions and Micelle, J Chem Theory Comput, 11 (2015) $340 \quad 5415-5425$.

341 [20] D.G. Leaist, Boltzmann Transformation of Taylor Dispersion Profiles to Determine 342 Concentration-Dependent Diffusion-Coefficients - Aqueous Cetylpyridinium Chloride near the 343 Critical Micelle Concentration, Ber Bunsen Phys Chem, 95 (1991) 113-117.

344 [21] R. Atkin, V.S.J. Craig, S. Biggs, Adsorption kinetics and structural arrangements of 345 cetylpyridinium bromide at the silica-aqueous interface, Langmuir, 17 (2001) 6155-6163.

346 [22] B. Lindman, M.C. Puyal, N. Kamenka, B. Brun, G. Gunnarsson, Micelle Formation of Ionic 347 Surfactants - Tracer Self-Diffusion Studies and Theoretical Calculations for Sodium Para348 Octylbenzenesulfonate, J Phys Chem-Us, 86 (1982) 1702-1711.

349 

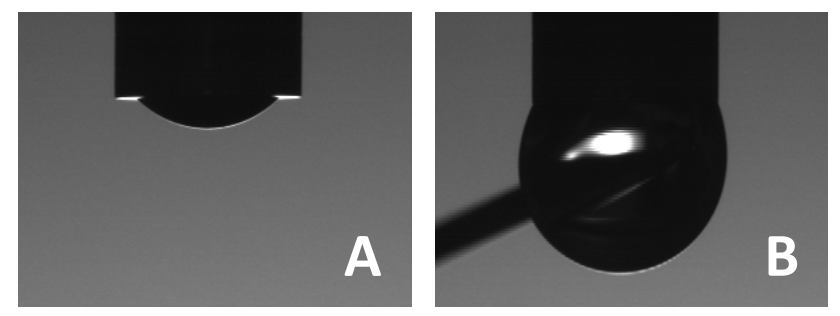

Drop Formation (A, B)
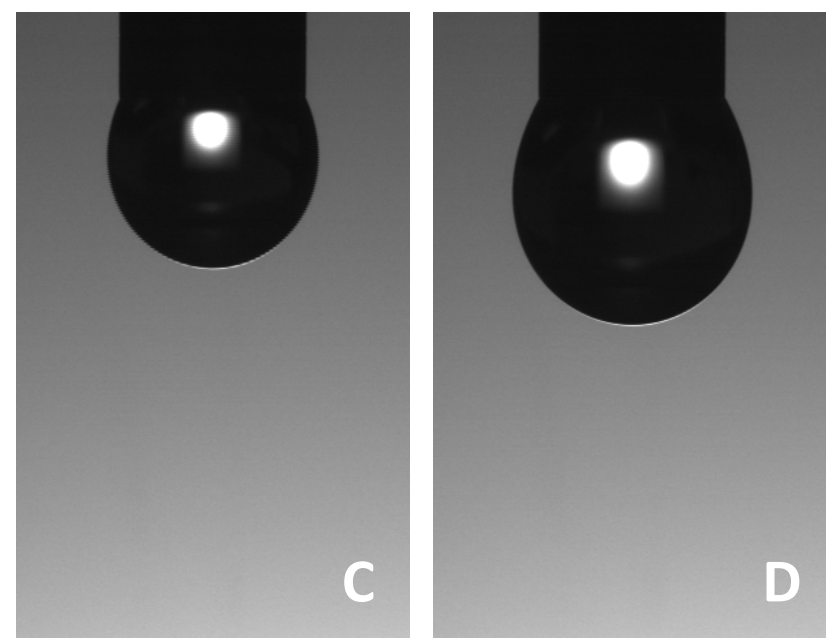

$\mathrm{t}=0 \mathrm{~min}$

$\mathrm{t}=1.3 \mathrm{~min}$

$\mathrm{V}=11.05 \mu \mathrm{L}$

$C_{\text {avg }}=1.82 \times 10^{-4} \mathrm{M}$

$\mathrm{C}_{\text {avg }}=3.12 \times 10^{-4} \mathrm{M}$

$\gamma=58.8 \mathrm{mN} / \mathrm{m}$

$\gamma=56.0 \mathrm{mN} / \mathrm{m}$

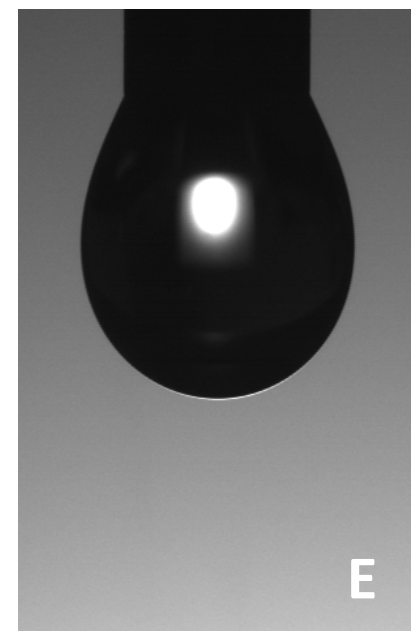

$\mathrm{t}=3.3 \mathrm{~min}$

$V=18.83 \mu \mathrm{L}$

$C_{\text {avg }}=1.07 \times 10^{-4} \mathrm{M}$

$\gamma=57.9 \mathrm{mN} / \mathrm{m}$

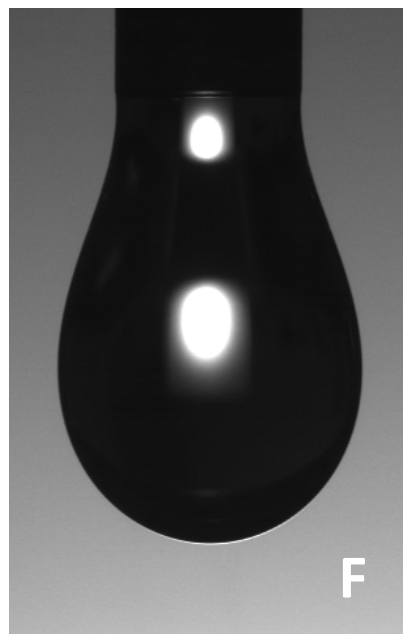

$\mathrm{t}=6.4 \mathrm{~min}$

$\mathrm{V}=31.15 \mu \mathrm{L}$

$C_{\text {avg }}=6.48 \times 10^{-5} \mathrm{M}$

$\gamma=61.2 \mathrm{mN} / \mathrm{m}$

Figure 1. Selected images from drop formation and expansion for aqueous CPC. Added CPC concentration $3.3 \times 10^{-4} \mathrm{M}$. Water flow rate $=3.87 \mu \mathrm{L} / \mathrm{min}$. 


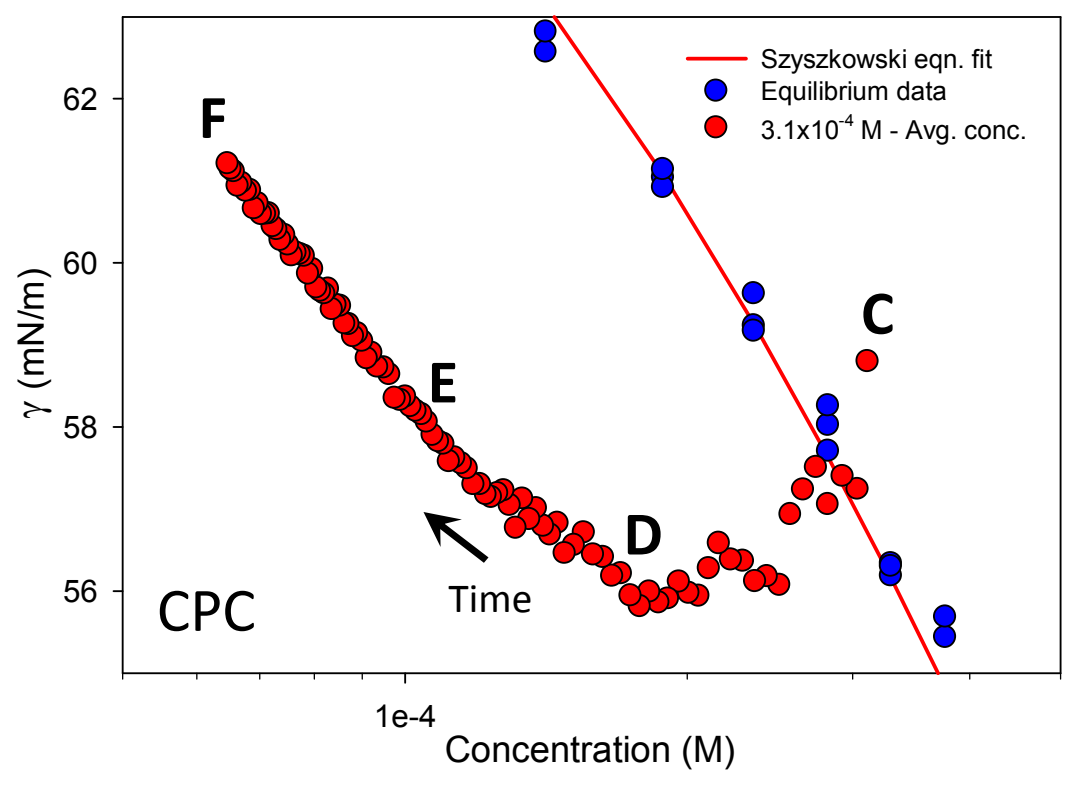

Figure 2. Measured surface tension $(\gamma)$ vs. average concentration in an expanding drop for the CPC system shown in Figure 1. Letters correspond to subfigures of the corresponding images in Figure 1. Equilibrium data and Szyszkowski equation fit to equilibrium data are also shown for comparison. 


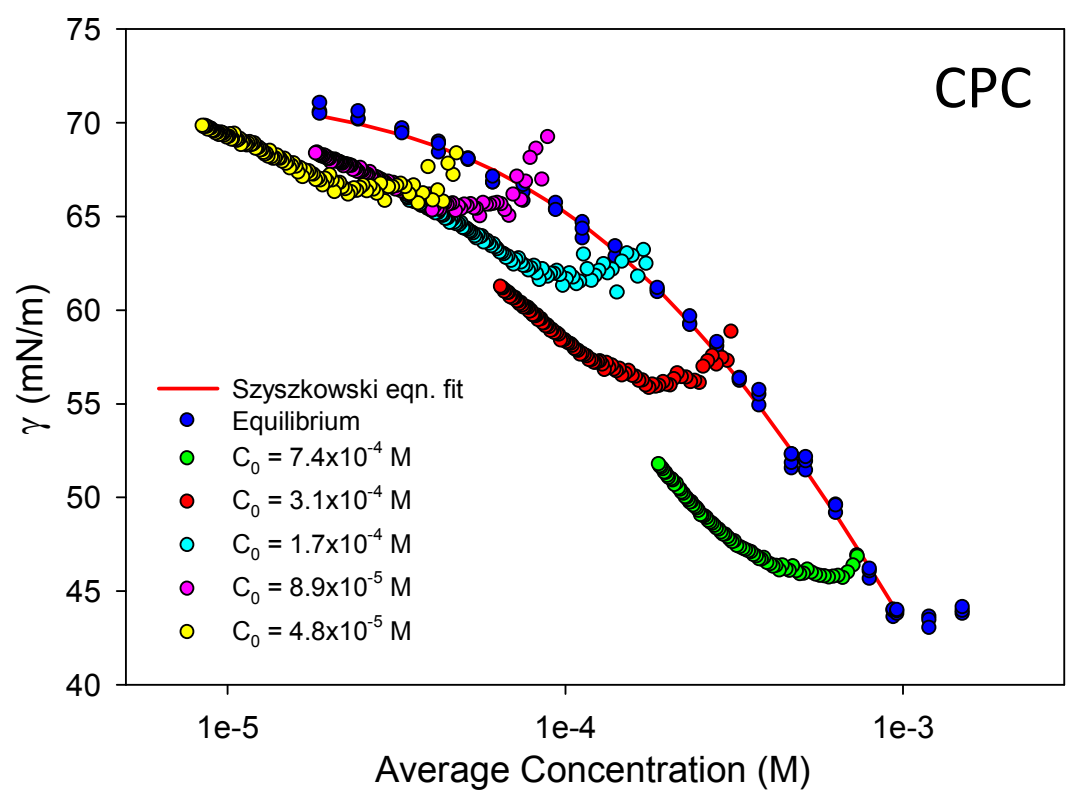

Figure 3. Measured surface tension $(\gamma)$ vs. average concentration in expanding drops for five different starting CPC concentrations. Equilibrium data and Szyszkowski equation fit to equilibrium data are also shown for comparison. 


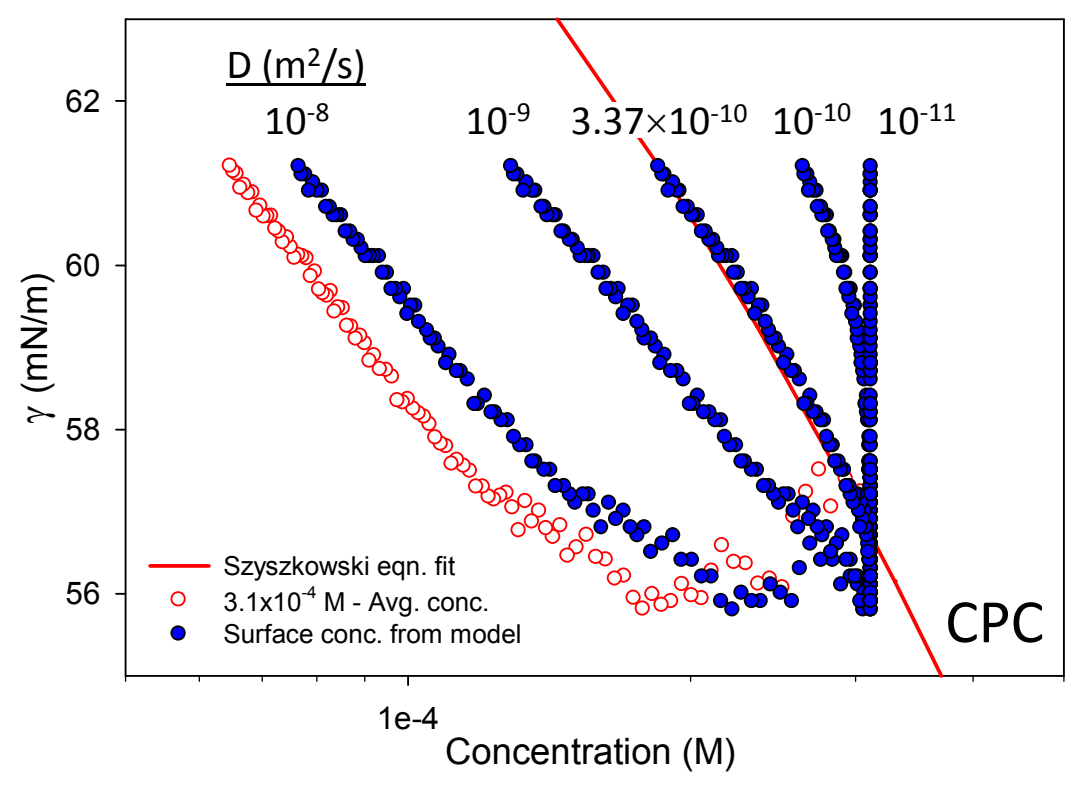

Figure 4. Measured surface tension $(\gamma)$ vs. calculated surface concentration in an expanding drop for the CPC system shown in Figures 1 and 2. Calculated surface concentrations are shown for different $D$ values. 


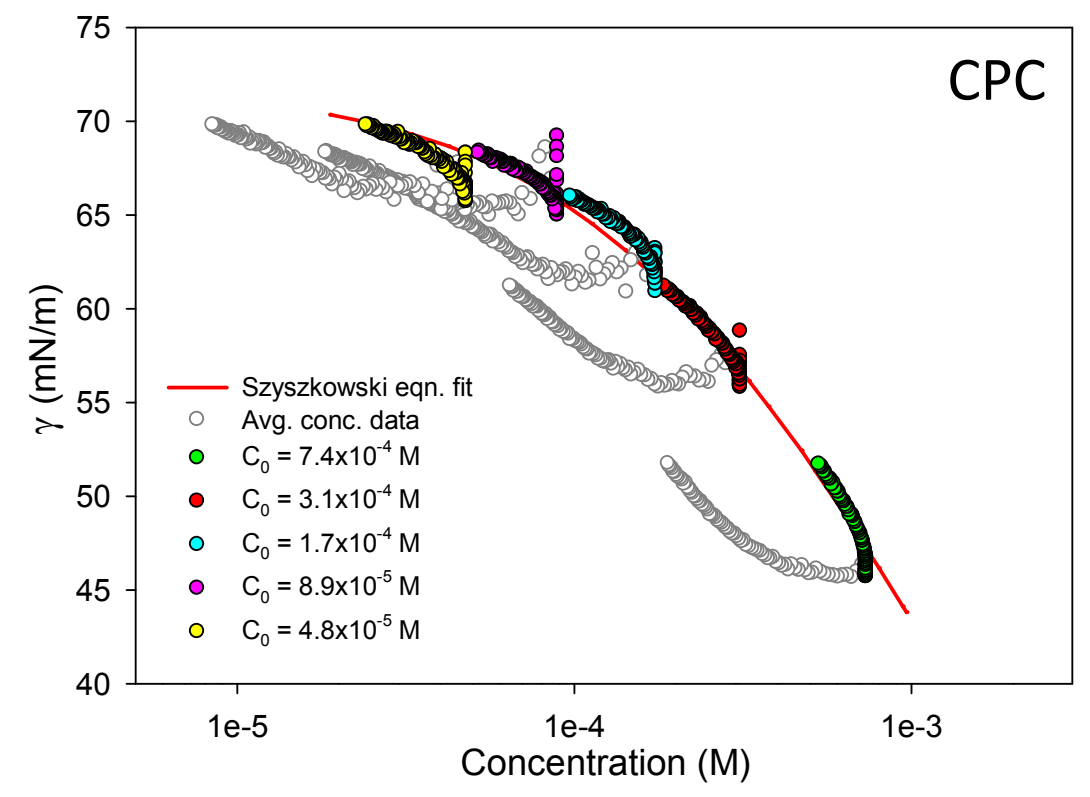

Figure 5. Measured surface tension $(\gamma)$ vs. calculated surface concentration in expanding drops for five different starting CPC concentrations. Calculated surface concentrations correspond to $D=3.37 \times 10^{-10} \mathrm{~m}^{2} / \mathrm{sec}$, the least-squares best-fit value for the data. 


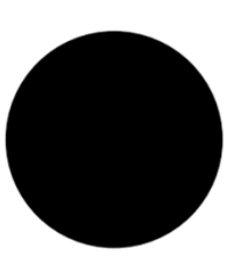

C

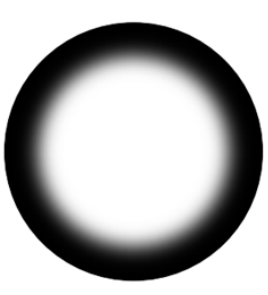

D

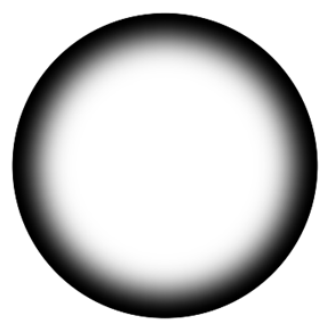

$\mathbf{E}$

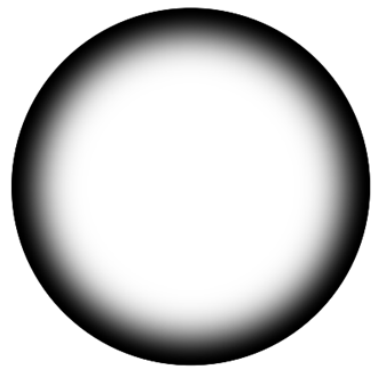

$\mathbf{F}$

Figure 6. Calculated spatial configuration of CPC for the system in Figures 1 and 2 , based on $D=3.37 \times 10^{-10} \mathrm{~m}^{2} / \mathrm{sec}$. Darker shading corresponds to higher concentration. (Black $=3.1 \times 10^{-4} \mathrm{M}$, White $=0 \mathrm{M}$ ). Letters correspond to drop images in Figure 1 and data points in Figure 2. 


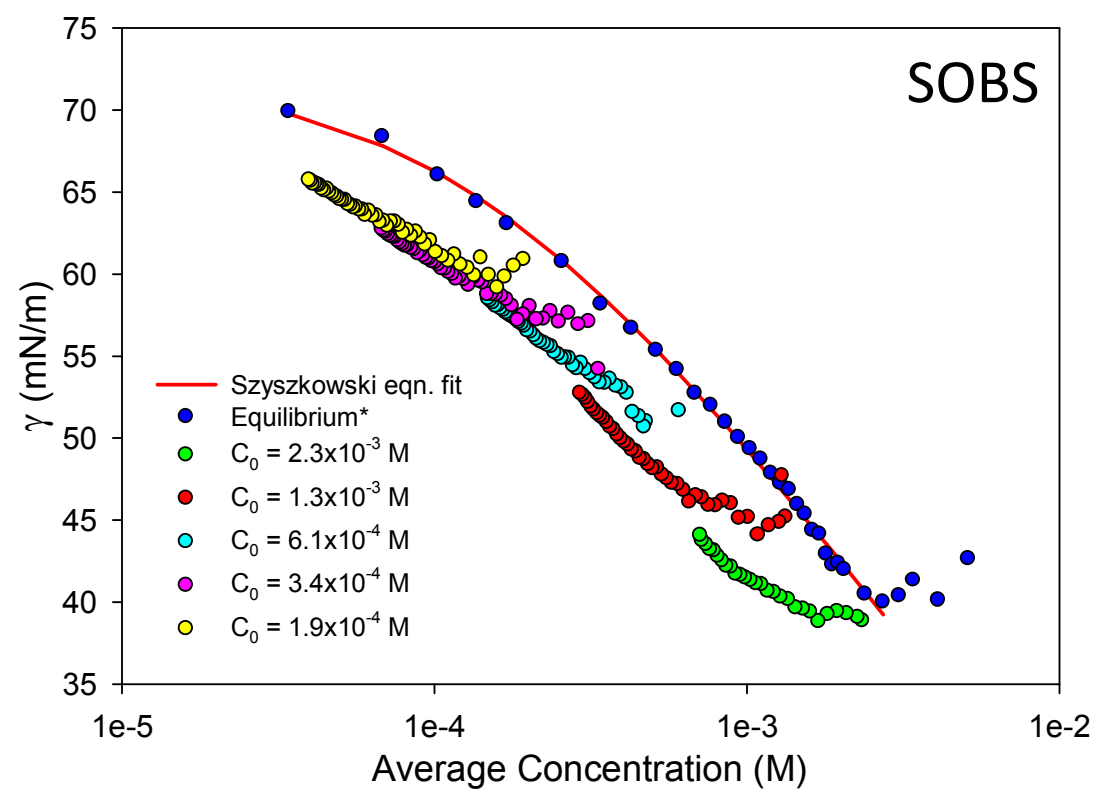

Figure 7. Measured surface tension $(\gamma)$ vs. average concentration in expanding drops for five different starting SOBS concentrations. Equilibrium data and Szyszkowski equation fit to equilibrium data are also shown for comparison. *Equilibrium data from Chen and Kibbey, 2006. 


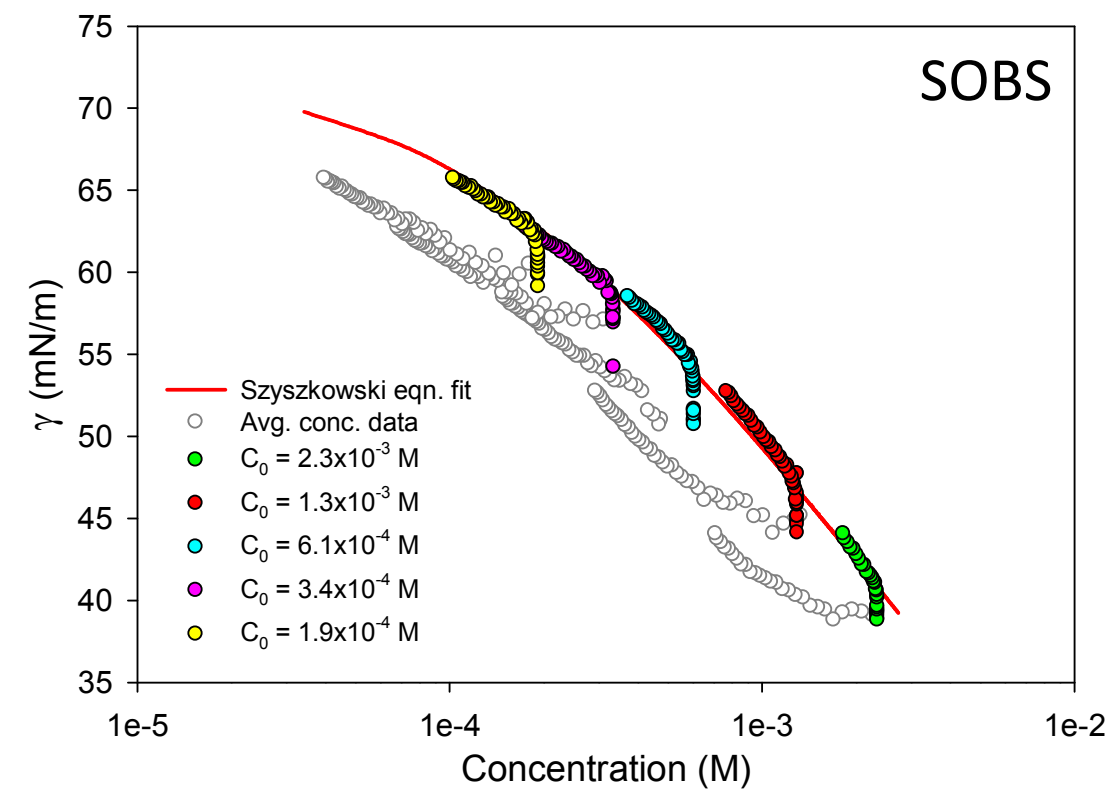

Figure 8. Measured surface tension $(\gamma)$ vs. calculated surface concentration in expanding drops for five different starting SOBS concentrations. Calculated surface concentrations correspond to $D=4.50 \times 10^{-10} \mathrm{~m}^{2} / \mathrm{sec}$, the least-squares best-fit value for the data. 


\section{Graphical Abstract}

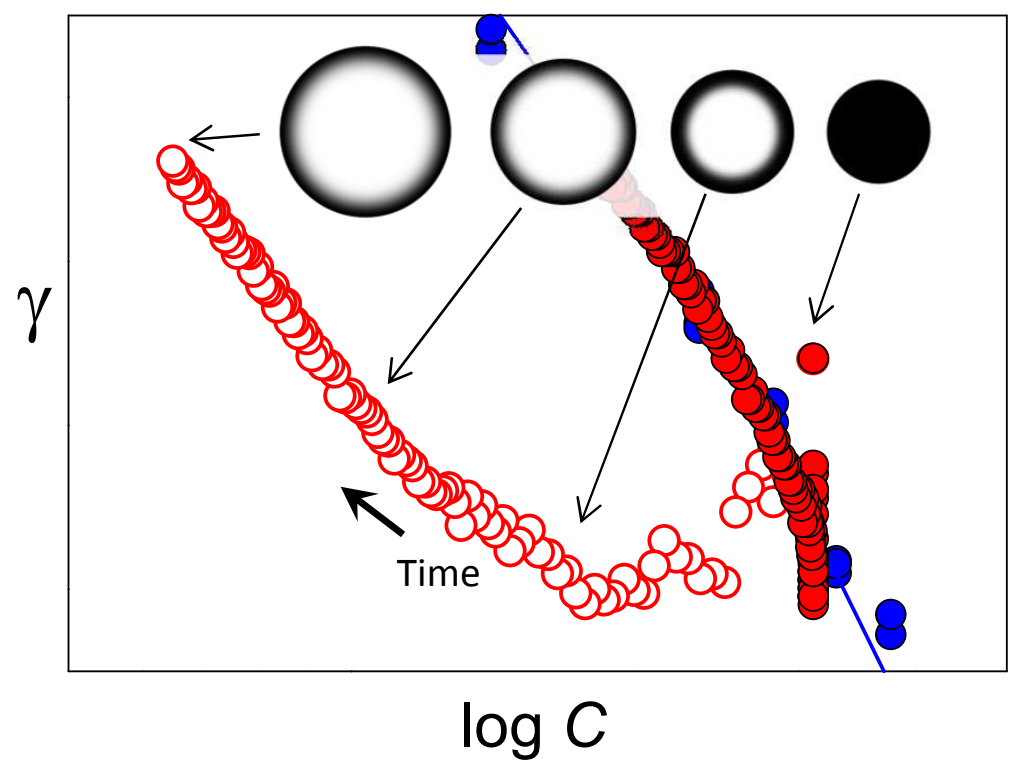

Surface tension was measured in drops of surfactant solution expanded by injection of surfactant-free water. Analysis with simulations suggests that molecular diffusion controls the dynamics of surface tension change for these systems. 